\title{
TTR
}

Traduction, terminologie, rédaction

\section{La traduction littéraire et le Canada : présentation}

\section{Nicole Côté, Danièle Marcoux et Madeleine Stratford}

Volume 28, numéro 1-2, 1er semestre-2e semestre 2015

La traduction littéraire et le Canada

Literary translation and Canada

URI : https://id.erudit.org/iderudit/1041653ar

DOI : https://doi.org/10.7202/1041653ar

Aller au sommaire du numéro

Éditeur(s)

Association canadienne de traductologie

ISSN

0835-8443 (imprimé)

1708-2188 (numérique)

Découvrir la revue

Citer ce document

Côté, N., Marcoux, D. \& Stratford, M. (2015). La traduction littéraire et le

Canada : présentation. TTR, 28(1-2), 133-138. https://doi.org/10.7202/1041653ar

Tous droits réservés @ Nicole Côté, Danièle Marcoux et Madeleine Stratford, 2017
Ce document est protégé par la loi sur le droit d'auteur. L'utilisation des services d'Érudit (y compris la reproduction) est assujettie à sa politique d'utilisation que vous pouvez consulter en ligne.

https://apropos.erudit.org/fr/usagers/politique-dutilisation/ 


\title{
La traduction littéraire et le Canada: présentation
}

\author{
Nicole Côté
}

Université de Sherbrooke

\author{
Danièle Marcoux \\ Université Concordia
}

Madeleine Stratford

Université du Québec en Outaouais

En 1977, Philip Stratford déclarait que le Canada n'avait pas de «tradition» de traduction littéraire, mais qu'une certaine «tendance» en ce sens commençait à poindre, en particulier depuis l'avènement du programme de financement du Conseil des arts du Canada. Près de quarante ans plus tard, il ne fait aucun doute que la traduction littéraire a maintenant une tradition et qu'elle a jusqu'ici joué un rôle-clé dans la formation identitaire du pays, sur les plans culturel, économique, politique, social, etc. Sa fonction d'agent de rapprochement entre les deux «solitudes» relève aujourd'hui de l'histoire ancienne. Bien quelle ait été nécessaire, cette conception semble désuète dans un pays comme le Canada qui, s'il conserve ses deux langues officielles, témoigne également d'une grande diversité ethnique, culturelle et linguistique, dont celle naguère niée des Premières Nations.

En effet, au cours des dernières décennies, une internationalisation et une diversification se dessinent au sein du polysystème canadien. Non seulement la scène littéraire s'est élargie grâce à une inclusion des littératures émergentes, minoritaires, migrantes, régionales, autochtones ou autres, mais elle s'est aussi enrichie grâce à un foisonnement de genres littéraires, sur support papier comme électronique. On traduit de plus en plus des littératures orales (slam, contes, chansons) et les littératures jeunesse, des genres hybrides comme le livre d'art. 
En outre, on traduit et on publie au Canada un nombre grandissant d'auteurs d'ailleurs, sans compter la popularité des auteurs canadiens plus traduits que jamais. Bref le dialogue littéraire entre les deux langues officielles s'est transformé en un échange pluriel résonnant des langues des Amériques, minoritaires ou non, si bien que le capital littéraire et traductif du Canada a aujourd'hui une portée planétaire. Témoin, l'inauguration du programme de résidence du Centre international de traduction littéraire de Banff, qui accueille chaque été des traductrices et traducteurs littéraires de partout dans le monde.

Dans un tel contexte, nous voulions établir un lien clair entre la traduction littéraire et le Canada (conjonction au sens fort) qui invite à la penser comme une constituante de la singularité canadienne, un lieu de synthèse de diverses influences, prolongement des cultures européennes, métissage avec les peuples autochtones, pluralité de langues, en plus des langues officielles. Le Canada est un pays traducteur s'inscrivant dans un continent traducteur. Par conséquent, il est intéressant de le lire comme l'héritier de traditions intellectuelles dont l'originalité est le fruit de la traduction. Les études en traductologie fournissent désormais un cadre de référence et d'analyse propice à la reconnaissance du rôle de la traduction littéraire dans l'éclosion, le maintien et la redéfinition de l'identité canadienne (mouvante, comme tout ce qui cherche à se perpétuer).

Ce numéro de TTR se veut une occasion de dresser un bilan des acquis et des perspectives d'avenir en matière de traduction et de traductologie littéraires. Les cinq articles inclus s'inscrivent dans deux axes complémentaires : la traduction au Canada d'œuvres littéraires d'origine étrangère et la diffusion de la littérature canadienne, ici comme ailleurs, au moyen de la traduction. Les trois premiers articles discutent de traduction de poésie, tandis que les deux autres abordent la traduction du vernaculaire, le premier dans un roman, le second dans diverses pièces de théâtre.

Dans l'article qui ouvre le numéro, «De Bellefeuille sous la plume de D. G. Jones», Patricia Godbout revisite les versions anglaises de Normand de Bellefeuille signées D. G. Jones, un des pionniers de la traduction de poésie québécoise vers l'anglais. Elle discute d'abord de la définition de la traduction que donne Arno Renken, pour qui elle est «un texte et un texte» (2012, p. 29), un texte original et une traduction, côte à côte, écrit Godbout, 
concluant préliminairement à une esthétique «éloignée d'un certain idéal de remplacement » chez D. G. Jones. Il s'agit pour Jones, aussi poète, d'accompagner les poèmes de langue française plutôt que de se substituer à eux. Godbout apparente ainsi cette approche à la non-traduction de Jacques Brault. Jones, Québécois d'adoption, était un interlocuteur de choix, avec son regard de l'intérieur sur la poésie québécoise, plus préoccupée, selon E. D. Blodgett, par le temps que par l'espace, leitmotiv de la littérature anglo-canadienne. Selon Godbout, la posture traductive de D. G. Jones rejette l'idée d'intraduisibilité et "revendique la liberté de "mal traduire", de jouer avec la matière donnée». Comme le dit l'auteur des originaux, de Bellefeuille, «ça ne revient jamais vraiment au Même»(1986, p. 22). Pourtant, la répétition, ou l'anaphore, est structurellement très présente dans les poèmes de Saint-Denys Garneau et serait selon Godbout quelque peu accentuée par Jones, puisque l'anglais s'en soucie moins. Nutting, qui a analysé la traduction, suggère que «son travail recèle une indéniable rigueur interne»(2006, p. 122), comme Godbout le recense. L'aspect méditatif des poèmes originaux de Bellefeuille est souligné en traduction, ce qui signifie aussi que Jones a fait passer cet aspect dans la traduction, lui qui a si bien su accompagner son poète.

Madeleine Stratford s'intéresse à la traduction du même classique québécois, Hector de Saint-Denys Garneau, mais étudie sa réception en espagnol, inscrivant la littérature québécoise dans une tradition latino-américaine. Elle s'attaque à une comparaison des traductions en espagnol de Saint-Denys Garneau, s'arrêtant particulièrement à deux poèmes en vers libres parmi les plus connus, "Accompagnement» et "Cage d'oiseau», et à leurs cinq traductions récentes. Considérant son étude comme une forme de "traductologie comparée», Stratford, après une analyse des poèmes sous leurs aspects formels (sonorités, répétitions lexicales, assonances et allitérations, longueurs des vers, mise en page, etc.), examine ces aspects dans les différentes versions espagnoles. Elle tient compte, dans les cas où la syntaxe a été modifiée, de la stylistique différentielle des langues. Par exemple, le pronom «je» en espagnol n'existant que pour mettre en relief, étant autrement inclus dans la conjugaison du verbe, son équivalent yo a, dans la plupart des traductions, été omis. Elle remarque que les répétitions structurelles et thématiques sont la plupart du temps respectées. L'attention particulière de Saint-Denys Garneau à la place des 
poèmes et à la mise en page, que Stratford attribue au fait qu'il était aussi peintre, n'est pas reproduite en traduction parce que « toutes les versions espagnoles des deux poèmes [...] ont été extirpées du "tout" dont elles faisaient originellement partie", certaines traductions, comme celle de Cohen, ne laissant plus aucun lien thématique subsister entre les deux poèmes. La mise en page, selon Stratford, résultera d'un compromis entre les contraintes du format éditorial et la mise en page originale, ces choix ayant une incidence "sur le rythme de lecture du poème, voire sur sa signification globale». Toutefois, Stratford en conclut que l'esthétique du poète demeure généralement en traduction.

Dans «Traduire au Québec la poésie étrangère: départager le rêve de la réalité», Mélanie Rivet rappelle qu'il se traduit au Québec de la poésie d'origine étrangère. Rivet discute de la traduction de poètes étrangers au Canada dans un contexte où la poésie possède un bassin de lecteurs passablement limité et où le système de subventions public soutient les Canadiens seulement. Son étude porte sur les poètes étrangers traduits au Québec entre 2000 et 2014. Elle répertorie les tendances quant aux langues et lieux d'origine des poètes traduits, les maisons d'édition qui les ont le plus traduits et les sources de financement disponibles pour leur publication. Une vingtaine de maisons d'éditions et particulièrement sept d'entre elles auraient publié en traduction près de 450 poètes étrangers. La plupart de ces poètes sur les 139 publiés en monographie vivent au Mexique. C'est donc l'espagnol qui remporte, et de loin, la palme des langues traduites, avec, très loin derrière, l'anglais. Ces poètes traduits jouissent déjà d'une grande reconnaissance dans leur pays. Les traducteurs sont habituellement liés à une maison d'édition, les plus nombreux étant affiliés aux Écrits des Forges (119/139). Un traducteur sur trois est aussi un ou une poète. Par ailleurs, aucun financement extérieur nétant accordé à la traduction de poètes étrangers, ce sont les maisons d'édition qui utilisent leurs propres fonds pour publier ces traductions. Rivet conclut qu'il existe une pratique bien établie de poésie étrangère au Québec par la traduction et que la plupart des poètes traduits viennent du Mexique, pays avec lequel les maisons d'édition - les Écrits des Forges principalement - maintiennent une pratique de coédition.

Dans «"These foreign c*nts've goat trouble wi the Queen's $f^{*}$ ckin English, ken”, ou ré-énoncer la voix scots de Trainspotting 
au moyen du Québec et de la (socio)linguistique», Alex Gauthier suggère que le français québécois familier est particulièrement apte à rendre les nuances du sociolecte scots présent dans Trainspotting. Gauthier discute de la traduction d'un vernaculaire scots en s'inspirant des prises de positions de Meschonnic, pour qui la traduction de la voix et du rythme devancent la traduction du sens. Gauthier examine longuement l'œuvre-phare d'Irvine Welsch (1993), le potentiel de subversion du sociolecte dans lequel il a couché son histoire de junkies d'Édimbourg, car ce scots, issu des tréfonds de la tradition écossaise, désormais parlé presque exclusivement par la classe ouvrière et les marginalisés, difficilement compréhensible au premier abord, sert à la fois d'affiche identitaire et de moyen de résistance à l'hégémonie anglo-britannique. Puis Gauthier examine les deux traductions hexagonales publiées (Lindor Fall en 1996 et Étienne en 2011) qui, selon lui, échouent justement parce qu'elles se préoccupent principalement du sens. Il faut dire que la traduction des sociolectes est un véritable cauchemar, ancrés qu'ils sont dans leurs territoires, histoires, classes sociales, ethnies. Gauthier expose ensuite sa solution: utiliser un certain français québécois qui, à l'instar du scots avec l'anglais britannique, marque davantage la distance avec le français normatif. Cette langue hors-norme, il la recrée à partir de «différences phonologiques et morphosyntaxiques du français québécois» (principalement montréalais) par rapport au français hexagonal, représentées graphiquement dans sa traduction. Selon Gauthier, ces différences «créent un système à plusieurs niveaux qui s'adapte à tous les protagonistes du roman et qui mène à une traduction visant à recréer les rapports de force exprimés par Welsh ainsi que l'impact de l'original sur le lecteur».

Enfin, Louise Ladouceur examine le surtitrage comme mode de traduction théâtrale simultanée dans les milieux francophones minoritaires. Vieux d'une trentaine d'année, ce mode de traduction d'abord employé à la Canadian Opera Company est désormais pratique courante au Canada francophone. Selon Ladouceur, "le théâtre étant un art vivant», les surtitres doivent s'ajuster à «la nature instable» du texte source livré sur scène et aux destinataires parlant une langue ou les deux. Le principal avantage du surtitrage est "qu'il permet à l'œuvre de rejoindre un public élargi tout en conservant sa spécificité linguistique et esthétique». L'auteure rappelle que le surtitrage se distingue de la traduction 
dramatique conventionnelle autant dans les moyens mis en ouvre pour produire le texte cible que dans sa réception. En effet, le surtitrage n'est pas issu d'une opération invisible et réservée aux seuls destinataires de la langue cible, la traduction s'affiche à tous les destinataires sans distinction, afin d'élargir le public cible. Le produit de la traduction dramatique est un texte autonome et stable, alors que celui des surtitres, ponctuel, fluctue pour autant "au gré des représentations». Ce faisant, note Ladouceur, ce théâtre bilingue "a pour effet de raviver l'insécurité linguistique des locuteurs de la langue minoritaire». Ce phénomène, précise Ladouceur, est absent des scènes anglophones, ce qui met en relief «l'asymétrie des langues officielles du Canada». Qui plus est, cette coexistence de l'original et d'une traduction impose une fidélité et une économie accrues face au public bilingue. Ladouceur traite donc non seulement de la spécificité du surtitrage comme mode de traduction dans le champ de la traduction audiovisuelle, mais aussi de certains enjeux théoriques et symboliques reliés à l'emploi des surtitres anglais dans les théâtres franco-canadiens.

Souhaitons que la lecture de ce numéro de TTR et des articles qui le composent montre à quel point les horizons de la traduction littéraire au Canada se sont élargis! En un demi-siècle à peine, elle est passée de tentative de dialogue entre «deux solitudes» pour devenir à présent une caisse de résonnance multiple entre des langues, des cultures et des genres littéraires, ici comme dans le monde entier. Dans les débats qui ébranlent les fondements identitaires des peuples, la traduction littéraire et le Canada inscrivent une voix exemplaire, digne d'être entendue.

\section{Références}

De Bellefeuille, Normand (1986). Catégoriques un deux et trois. TroisRivières, Écrits des Forges.

Nutting, Stephanie (2006). «On D. G. Jones and Translating Outside». In A. Whitfield, dir. Writing Between the Lines. Waterloo, Wilfrid Laurier Press, p. 107-138.

Renken, Arno (2012). Babel heureuse. Pour lire la traduction. Paris, Van Dieren Éditeur, coll. «Par ailleurs».

Stratford, Philip (1977). Bibliographie de livres canadiens traduits de l'anglais au français et du français à l'anglais/Bibliography of Canadian Books in Translation: French to English and English to French. $2^{\mathrm{e}}$ édition, Ottawa, Conseil canadien de recherches sur les humanités/ Humanities Research Council of Canada. 